\title{
FireWatch: G.I.S.-assisted Wireless Sensor Networks for Forest Fires
}

\author{
Panayiotis G. Andreou, George Constantinou, Demetrios Zeinalipour-Yazti, \\ and George Samaras \\ Department of Computer Science, University of Cyprus, Nicosia, Cyprus \\ \{panic, gconst02, dzeina, cssamara\}@cs.ucy.ac.cy
}

\begin{abstract}
Traditional satellite and camera-based systems, are currently the predominant methods for detecting forest fires. Our study has identified that these systems lack immediacy as detected fires must gain some momentum before they are detected. In addition, they suffer from decreased accuracy especially during the night, where visibility is diminished. In this paper, we present FireWatch, a system that aims to overcome the aforementioned limitations by combining a number of technologies including Wireless Sensor Networks, Computer-supported Cooperative Work and Geographic Information Systems in a transparent manner. Compared to satellite and camera-based approaches, FireWatch is able to detect forest fires more accurately and forecast the forest fire danger more promptly. FireWatch is currently scheduled to be deployed at the Cypriot Department of Forests.
\end{abstract}

\section{Introduction}

Traditional satellite and camera-based systems $[4,5]$, such as MODerate resolution Imaging Spectroradiometer (MODIS) [5], are currently the predominant methods for detecting forest fires. These systems have the ability to predict global changes in order to assist organizations in making sound decisions concerning the protection of our environment from natural disasters, including fires. Camera-based systems have been popular, especially in Europe [2-4], during the past few years. These systems usually consist of terrestrial, tower-based cameras that enable reliable and automated early detection of fires. In most cases, these systems are supplemented by alarm mechanisms that alert the involved users of potential risks thus supporting the final decisions on further actions. Although camera-based systems provide a more fine-grained solution than satellite systems, because of the smaller scanning regions and the continuous human monitoring factor, they lack immediacy as detected fires must gain some momentum before these are detected. In addition, camera-based systems suffer like their satellite-based counterparts from decreased accuracy, especially during the night where visibility is diminished. Both satellite and camera-based systems rely mainly on the human factor to analyze the findings and decide on the course of action. 


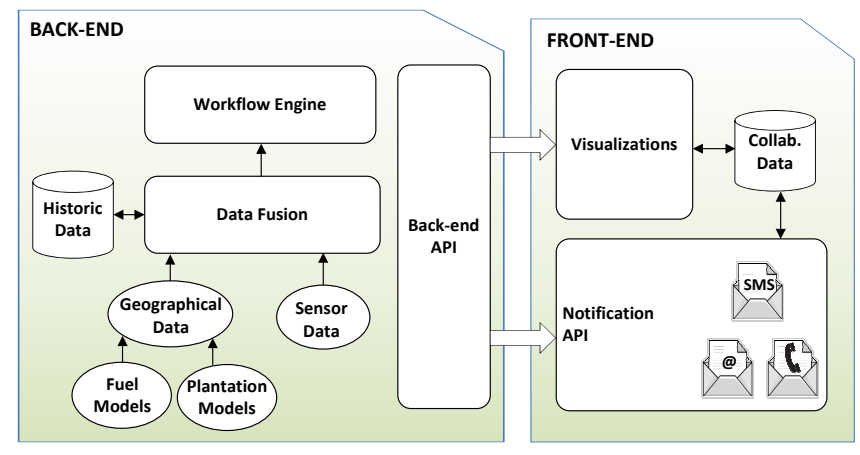

Fig. 1. The FireWatch architecture.

In this paper, we present the FireWatch system ${ }^{1}$ that aims to overcome the aforementioned limitations by combining a number of technologies including Wireless Sensor Networks (WSNs), Computer-supported Cooperative Work (CSCW) and Geographic Information Systems (GIS). The Wireless Sensor Network incorporates energy efficient algorithms that continuously monitor the environment and provide real-time measurements. The Collaboration Engine employs both snapshot and historic fire detection/prediction algorithms and enables the notification of all involved authorities. The enhanced Geographic Information System, besides managing and presenting all types of geographical data, inserts an additional layer that displays plantation and fuel models thus enabling fire officers to better assess the situation.

Compared to satellite and camera-based approaches, FireWatch is able to detect forest fires more accurately and forecast the forest fire danger more promptly. As a result, Cypriot forest officers, who are currently relying on watch towers and periodic patrolling of the forest, will be provided with more accurate decision-support data, hence they will plan a more refined course of action.

\section{System Architecture}

An overview of the FireWatch architecture is illustrated in Figure 1. It consists of two tiers, the Back-end, which is responsible for data collection, data analysis and event detection/prediction, and the Front-end, which visualizes the data as well as provides the means to contact appropriate authorities (e.g., voice messages, sms, email) in the case of a fore fire detection. We now provide more detail on each component of the FireWatch architecture.

- The Sensor Data component, which stores the real-time data provided by the wireless sensor network deployed in a high risk forest area. It incorporates

\footnotetext{
${ }^{1}$ The FireWatch System, http://firewatch.cs.ucy.ac.cy/
} 

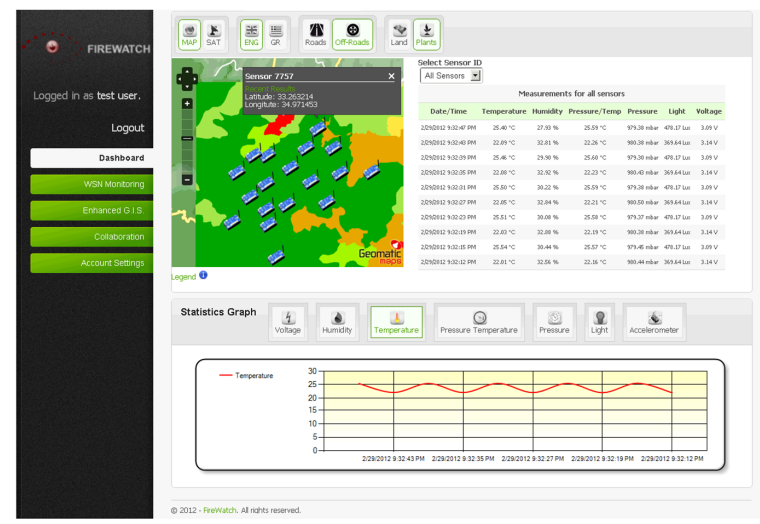

\section{Landuse}

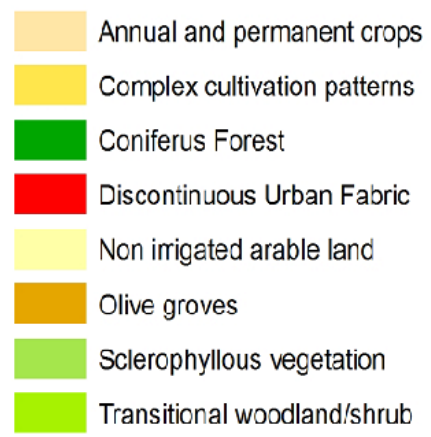

Fig. 2. The FireWatch interface.

energy-efficient algorithms [1], which run locally at each sensor node in order to increase the network longevity and minimize maintenance costs.

- The Geographical Data component, which stores geographical information as well as road and off-road data. Furthermore, it incorporates fuel and plantation models for estimating fire behavior.

- The Data Fusion component, which correlates sensor and geographical data and produces a comprehensive view of the network status. Additionally, this data is stored in a database (Historic Data), in order to by analyzed together with other data utilized by the organization (e.g., meteorological data).

- The Workflow Engine, which incorporates information models that analyze the real-time and historic data so as to detect/predict forest fire events.

- The Visualizations component displays all data in a visual manner (i.e., map view, tabular format, graphs). Additionally, it provides different interfaces for querying the data of each component.

- The Notification API support different notification mechanisms for providing alerts to users (e.g., visual alerts, audio alerts) and involved authorities (e.g., voice messages, SMS, Email).

FireWatch adopts a virtualization paradigm in order to increase flexibility in deployment and provide a dynamic, scalable and robust platform. It incorporates three individual virtual machines that can be maintained independently. The first virtual machine manages the wireless sensor network and includes tools for porting SQL queries to sensor nodes. Additionally, it provides APIs for translating raw measurements to real values. The second virtual machine consists of a GIS server that provides geographical data to the platform. It includes a map visualization component as well as APIs for creating/updating map layers. The third virtual machine comprises the collaboration system. It maintains a dedicated workflow engine where users can create new workflows or update existing ones according to organizational requirements. 


\section{$3 \quad$ FireWatch Interface}

FireWatch features an innovative web-based interface (see Figure 2) that enables fire officers to overview the status of the network as well as query/retrieve specific information. The FireWatch interface is composed of the following primary visualization components:

- Dashboard: Provides an overview of the whole system. Users can observe the latest sensor measurements both in tabular format and charts. Additionally, a map of the high risk forest area and the sensor locations is displayed.

- WSN Monitoring: Enables users to execute queries in order to monitor and access real-time and historic sensor measurements (e.g., temperature, humidity, etc.). WSN health and predicted lifetime is also available.

- Enhanced G.I.S.: Plots sensor locations on the map as well as provides alerts to users about possible fire detection/prediction events. The map component supports three modes: i) normal view; ii) satellite view; and iii) plantation/fuel models view. Additionally, in the event of a forest fire, it can provide alternative off-road routes to the location of the event.

- Collaboration: FireWatch users can search and retrieve appropriate government authorities that can aid in forest fire management. A number of different communication techniques such as alerts, sms, emails are available.

\section{Conclusions}

In this paper, we have presented FireWatch, a novel fire detection/prediction platform that combines technologies from the areas of Wireless Sensor Networks, Computer-supported Cooperative Work and Geographic Information Systems under a uniform framework. FireWatch virtualization mechanisms increase flexibility in deployment and provide a dynamicity, scalability and robustness. Finally, its multi-modal interface enables users to acquire a holistic view of the network, take informed decisions and coordinate easily with involved authorities.

Acknowledgements This work is partly supported by the European Union under the project CONET (\#224053), the project FireWatch (\#0609-BIE/09) sponsored by the Cyprus Research Promotion Foundation, and the third author's startup grant sponsored by the University of Cyprus.

\section{References}

[1] Andreou, P., Zeinalipour-Yiazti, D., Chrysanthis, P.K., Samaras, G.: Towards a Network-aware Middleware for Wireless Sensor Networks. 8th International Workshop on Data Management for Sensor Networks (DMSN'11), The Westin Hotel, Seattle, WA, USA, August 29, (2011)

[2] European Commision Joined Research Center: Forest Fires in Southern Europe. Report No.1, July (2001)

[3] European Forest Fire Information System EFFIS: http://effis.jrc.it/Home/

[4] Hochiki Europe: http://www.hochikieurope.com/

[5] NASA MODIS website: http://modis.gsfc.nasa.gov/ 